\title{
固体核磁共振中三能级体系的 $180^{\circ}$ 组合脉冲
}

\author{
李鲗颖 王东生 㿟学文
}

(华东师范大学物理系, 上海)

在脉冲傅里叶变换核磁共振中, 射频激发脉冲的谱宽一直是个重要的问题. 近年来发展 起来的组合脉冲激发 ${ }^{[1-3]}$, 在一定程度上解决了上述问题, 广泛用于核自旋的布居数反转、宽 带去耦、多量子选择激发上。但在固体核磁共振中，组合脉冲的文献却不多见。最近，Tycko 等人 ${ }^{[4]}$ 用平均哈密顿理论提出了一个固体核磁共振中的 $180^{\circ}$ 组合脉冲: $45_{x}^{\circ} 180_{y}^{\circ} 90^{\circ} 180_{y}^{\circ} 45_{x}^{\circ}$; Levitt ${ }^{[j]}$ 等人也提出了如何获得 $90^{\circ}$ 组合脉冲的方法, 使得固体核磁共振中组合脉冲的研究活 跃起来。在固体样品中, 特别是 ${ }^{1} \mathrm{H}$ 和 ${ }^{2} \mathrm{H}$ 这两种常见核中存在很强的偶极与四极相互作用. 而射频场强度由于技术上的困难不能做到很强, 使射频场强度远大于核自旋体系内部的相互 作用这一条件不能得到满足。这样,射频场对核自旋体系的作用就不能看成是理想的转动,不 能达到均勺放发的目的. 用组合脉冲对核自旋体系进行激发, 就是要在脉冲作用期间将体系 内部的相互作用的影响消除掉, 使脉冲的作用仍可看成是理想的转动, 从而达到均匀激发的目 的. 本文给出一个固体三能级体系的 $180^{\circ}$ 组合脉冲:

$$
R_{x}\left(180^{\circ}\right)-130_{x}^{\circ} 80_{x}^{\circ} 40_{x}^{\circ} 130_{y}^{\circ} 80 \% 40 \% 130_{x}^{\circ} 80_{z}^{\circ} 40_{x}^{\circ} \text { 。 }
$$

它的效果很好, 可以用于 ${ }^{2} \mathrm{H}$ 核的弛豫时间的测量上。

\section{一、计算机模拟分析}

在用计算机进行计算时, 是按照核自旋体系的密度矩阵遵循的 Master 方程的一般解进行 的 ${ }^{(6)}$, 即

$$
\sigma(t)=T \exp \left[-i \int_{0}^{t} \mathscr{C}^{\mathscr{C}}\left(t^{\prime}\right) d t^{\prime}\right] \sigma(0)\left\{T \operatorname { e x p } \left[-i \int_{0}^{t} \mathscr{Y}^{\left.\left.\mathscr{C}\left(t^{\prime}\right) d t^{\prime}\right]\right\}^{-1},}\right.\right.
$$

其中 $T$ 是 Dyson 时序算符. 对于 $I=1$ 核,体系总哈密顿算符为 ${ }^{[6]}$

$$
\mathscr{C}(t)=\mathscr{E}_{H}(t)+\mathscr{K}_{Q}+\mathscr{C}_{D}+\mathscr{E} \mathscr{C}_{\text {off }},
$$

这里 $\mathscr{Y}_{i f}(t)$ 是射频场与核体系的相互作用; $\mathscr{C}_{Q}, \mathscr{C}_{D}$ 是四极与偶极相互作用; $\mathscr{C}_{\text {off }}$ 是 包括化学位移在内的偏离共振作用. 一般情况下, $\mathscr{U}_{Q} \gg \mathscr{C}_{D}, \mathscr{Y}_{\text {off }}$, 所以, $\mathscr{C}(t)=$ $\mathscr{E}_{r}(t)+\mathscr{E}_{0}$.

先设初始时体系处于热平衡状态, 即 $\sigma(0)$ 正比于 $I_{s}$, 然后求出脉冲作用结束后的密度 矩阵 $\sigma_{f}$ 来, 最后组合脉冲的反转效果用 $-\left\langle I_{s}\right\rangle=-\operatorname{Tr}\left[I_{s} \sigma_{f}\right] / \operatorname{Tr}\left[I_{s}^{2}\right]$ 来表示. 计算的程序 包括用数值方法将体系的总哈密顿算符对角化.

图 1 是计算机按上述方法对 (A) $180^{\circ}$ 单脉冲, (B) $45_{x}^{\circ} 180_{y}^{\circ} 90^{\circ} 180^{\circ} 45_{*}^{\circ}$ 和 (C) $R_{x}\left(180^{\circ}\right)$

本文1986 年11月13日收到. 


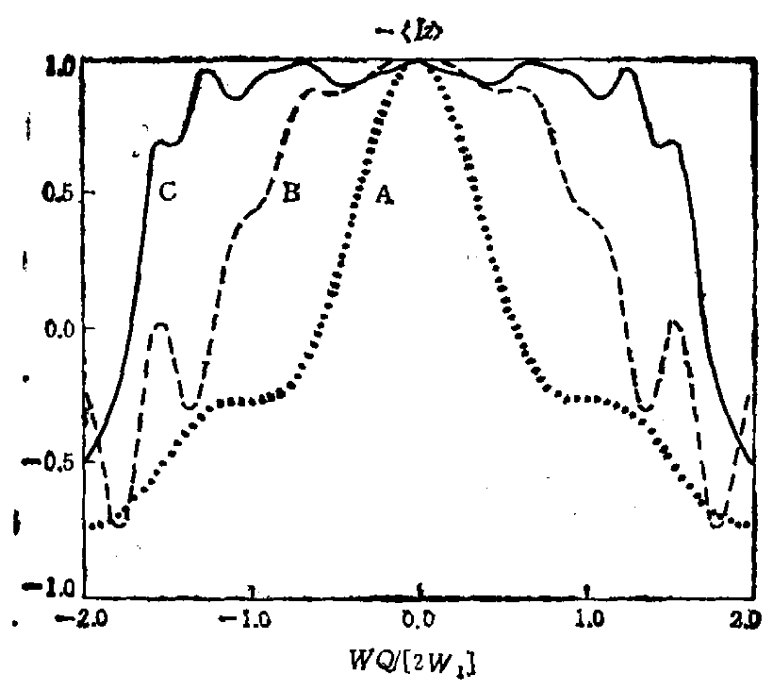

图 1 计算机的模拟曲线
A. $180 ;(\ldots . . .$.$) ;$
B. $45 ; 180 ; 90 ; 180 ; 45_{*}^{\circ}(--)$,
C. $R_{x}\left(180^{\circ}\right)(\longrightarrow$

模拟计算的结果. 横轴是参数 $W_{\mathrm{Q}} / 2 W_{1}$, 纵轴是反映反转效果的一 $\left\langle I_{x}\right\rangle$. 从图中可以看出, $R_{x}\left(180^{\circ}\right)$ 的激发谱宽比 $180^{\circ}$ 单脉冲大得多，并且也超过了五脉冲 (B). 若以一〈Is $\rangle$ 下降 $20 \%$ 为界, 对单脉冲, $\left|W_{Q} / 2 W_{1}\right|<0.25$; 对五脉冲 $45_{x}^{\circ} 180_{y}^{\circ} 90_{2}^{\circ} 180_{y}^{\circ} 45^{\circ}$, 有 $\left|W_{Q} / 2 W_{1}\right|<0.75$; 而对 $R_{x}\left(180^{\circ}\right)$, 则有 $\left|W_{Q} / 2 W_{1}\right|>1.3$. 因此, $R_{x}\left(180^{\circ}\right)$ 对三能级体系的布居数反转效果 很好.

\section{二、实验与结果}

为验证组合脉冲的反转效果，采用图 2 所示的脉冲序列。为克服仪器的死时间， $180^{\circ}$ 脉 冲后接用组合脉冲构成的固体回波序列 ${ }^{[3]}$, 在实验中的射频场强度下，回波引起的谱线失真较 小, 而主要失真是由前面的 $180^{\circ}$ 单脉冲或组合脉冲造成的.

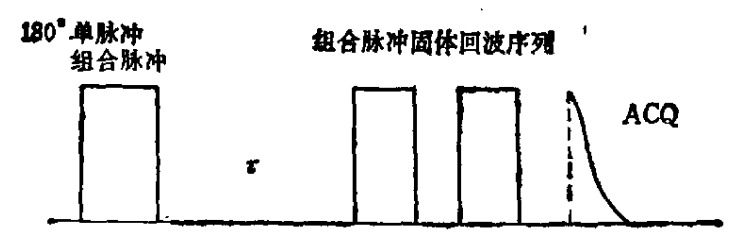

图 2 实验中采用的脉冲序列 后面的是组合脉冲固体回波序列[s]

实验是在 MSL-300 谱仪上做的, 样品是気代有机玻玹粉末。氛核共振频率是 46.071 $\mathrm{MHz}$ ，实验中射频场强度 $W_{1} / 2 \pi-31.25 \mathrm{KHz}$ ，对应 ${ }^{2} \mathrm{H}$ 核的 $90^{\circ}$ 脉冲宽度是 $8.0 \mu \mathrm{s}$ 。

图 3 是实验结果. 其中图 (3A) 是只用回波序列作的谱，这里将它作为标准谱. 图 (3B) 是 $180^{\circ}$ 单脉冲得到的结果, 从谱图中可看到,它存在着严重失真, 在频率超出 $\pm 20 \mathrm{KHz}$ 以外 基本上没得到激发。图 (3C) 是 $45_{x}^{\circ} 180_{y}^{\circ} 90^{\circ} 180 \% 45_{x}^{\circ}$ 组合脉冲得到的谱，与单脉冲相比有明 显的改善. 但由于激发谱宽仍不够，使在频率 $\pm 40 \mathrm{kHz}$ 以外仍有失真. 图 (3D) 是本文提出 的 $R_{x}\left(180^{\circ}\right)$ 组合脉冲得到的谱. 它比前两个脉冲有宽得多的激发谱宽, 得到与标准谱差不 多的线型,谱线在 $\pm 40 \mathrm{kHz}$ 附近的形状很清楚。 


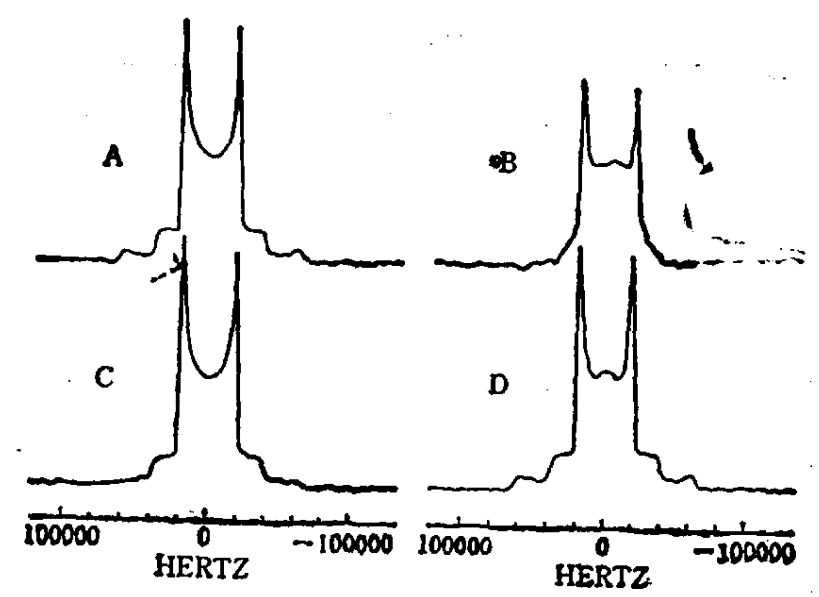

因 3 用图 2 脉冲序列得到的㲴代有机玻璃粉末样品的 NMR 徣
A. 只用回诐序列作的谓；
B. $180^{\circ}$ 单眿冲;
C. $45 ; 180 ; 90 ; 180 ; 45 *$ 组合脉冲;
D. $R_{n}\left(180^{\circ}\right)$ 组合脉冲

\section{三、讨 论}

从上面的计算机模拟与实验结果可看到, 本文提出的 $\boldsymbol{R}_{\boldsymbol{x}}\left(180^{\circ}\right)$ 组合脉冲有很宽的激发 谱宽，不仅大大优于 $180^{\circ}$ 单脉冲，而且也超过了文献 [4] 给出的组合脉冲. 计算分析与实验 结果是一致的. 用组合脉冲代替传统的单脉冲，在很大程度上克服了有限脉冲宽度的影响造 '成的困难. 本文提出的 $R_{x}\left(180^{\circ}\right)$ 组合脉冲,能使三能级体系的布居数有效反转，可用于 ${ }^{2} \mathrm{H}$ 核的驰豫时间的测量中。

上面的结论同样地适用于自旋 $l-1 / 2$ 双核体系。

\section{* * 文献}

[ 1 ] Tycko, R, Plyr. Rev. Letw, 51(1983), 9: 775.

[2] Counsell, C, Levitt, M. H. and Ernst, R. R., J. Magn. Reson, 63(1985), 133.

[ 3 ] Tyeko, R., Cho, M. H., Schneider, E. and Pines, A.,.J. Magn. Reson. 61(1985), 90.

[4] Tycko, R., Schneider, E. and Pines, A., J. Chem. Phys, 81(1984), 2: 680.

[ 5] Levitt, M. H., Suter, D. and Ernst, R. R., J. Chem. Phys., 80(1984), 7: 3064.

[6] Mehring, M. Principles of High Resolution NMR in Solids, Springer, Berlin-Heidelberg-New York, 1983. 